\title{
Production of highly concentrated oil-in-water emulsions using dual-channel microfluidization: Use of individual and mixed natural emulsifiers (saponin and lecithin)
}

Yihui Deng ${ }^{\mathrm{a}, \mathrm{b}}$, Yanyan Zhou ${ }^{\mathrm{c}}$, Long Bai ${ }^{\mathrm{b}}$, Fuguo Liu ${ }^{\mathrm{b}}$, Ruojie Zhang ${ }^{\mathrm{b}}$, Zipei Zhang ${ }^{\mathrm{b}}$, Bingjing Zheng ${ }^{\mathrm{b}}$,jing Zhengg, ${ }^{*, a}$, David Julian McClements*,

\author{
${ }^{a}$ College of Pharmacy, Shenyang Pharmaceutical University, 103 Wenhua Road, \\ Shenyang, Liaoning 110016, China \\ ${ }^{\mathrm{b}}$ Biopolymers and Colloids Laboratory, Department of Food Science, University of \\ Massachusetts, Amherst, MA 01003, USA \\ ${ }^{c}$ Research and Development Center, Zhejiang Medicine Co., Ltd Xinchang \\ Pharmaceutical Factory, Shaoxing, Zhejiang 312500, China
}

Journal: Food Research International

Submitted: December 2016; Revised: January 31, 2017; Re-Revised: February 2017

*Corresponding author: Yihui Deng, College of Pharmacy, Shenyang Pharmaceutical University, 103 Wenhua Road, Shenyang, Liaoning 110016, China; E-mail: dengyihui@syphu.edu.cn; Phone: +86 (0) 24 23986316; Fax: +86 (0) 2423986316.

*Corresponding author: David Julian McClements, Department of Food Science, University of Massachusetts Amherst, Amherst, MA 01003, USA; E-mail: mcclements@foodsci.umass.edu; Phone: +1 413545 1019; Fax: +1 4135451262 


\section{Abstract}

The fabrication of concentrated oil-in-water emulsions is useful for reducing storage and transportation costs, as well as for providing desirable textural, optical, stability, and release characteristics in commercial products. In this study, 50 wt\% oil-in-water emulsions were produced from natural emulsifiers using high-pressure dual-channel microfluidization (89.6 MPa, 1 pass). The particle size and charge characteristics of emulsions stabilized using a hydrophilic biosurfactant (quillaja saponin) or mixtures of hydrophilic and hydrophobic biosurfactants (quillaja saponin + soy lecithin) were measured. The physical stability of the emulsions was determined during storage under quiescent conditions $\left(\mathrm{pH} 7,25^{\circ} \mathrm{C}\right)$. The mean droplet diameter and polydispersity decreased with increasing hydrophilic and hydrophobic biosurfactant concentration. Surface potential measurements indicated that interfacial composition depended on the amount of hydrophilic and hydrophobic biosurfactant present. The inclusion of hydrophobic emulsifier in the oil phase and hydrophilic emulsifier in the aqueous phase prior to homogenization, led to the formation of smaller oil droplets than using the hydrophilic emulsifier alone. The relatively small size and polydispersity of the droplets in the mixed-emulsifier systems led to a higher emulsion viscosity and a better aggregation stability, i.e., there was a smaller change in particle size during storage. However, some creaming was still observed in the emulsions due to the presence of a fraction of relatively large droplets. In summary, concentrated emulsions stabilized by mixed biosurfactants may be advantageous for commercial application in certain food, beverage, and pharmaceutical products.

Keywords: emulsions; mixed surfactants; natural emulsifiers; biosurfactants; dual-channel microfluidization; physical stability; homogenization 


\section{Introduction}

Oil-in-water emulsions are typically fabricated in the food industry by homogenizing an oil and aqueous phase together in the presence of an emulsifier using mechanical devices, such as high shear mixers, colloid mills, high pressure valve homogenizers, microfluidizers, and sonicators (Friberg, Larsson, \& Sjoblom, 2003; McClements, 2015; Schubert \& Engel, 2004). The production of dilute emulsions containing fine droplets is often relatively straightforward, but for certain applications it is advantageous to produce highly concentrated emulsions $(\geq 50 \%)$. The appearance, texture, flavor profile, and stability of emulsions is influenced by their droplet concentration, e.g., emulsion viscosity, lightness, flavor retention, and creaming stability all tend to increase with increasing droplet level (McClements, 2015). Consequently, food manufacturers often need to produce concentrated emulsions so as to obtain the quality attributes expected in a particular product, such as the "creaminess" of creams, sauces, salad dressings, and mayonnaise. Studies have shown that the rate of lipid oxidation in oil-in-water emulsions is reduced when the total oil level is increased (Osborn \& Akoh, 2004), and so increasing droplet concentration may improve the shelf-life of chemically labile products. The fabrication of highly concentrated emulsions may also be useful to reduce storage and transportation costs (Lissant, 1966; Piorkowski \& McClements, 2014). In this case, a concentrated emulsion is initially created, which is then diluted when it is applied in the final product. Finally, concentrated emulsions may be used to increase the loading capacity of emulsion-based delivery systems for hydrophobic bioactives (Muller, Harden, \& Keck, 2012). The ability to produce concentrated emulsions containing small droplets depends on homogenizer type and operating conditions, as well as the nature and concentration of emulsifiers used (Jafari, Assadpoor, He, \& Bhandari, 2008; Walstra, 1993). In particular, the droplet collision frequency tends to increase with increasing 
droplet concentration, leading to a greater extent of droplet coalescence within the homogenizer (Gupta, Eral, Hatton, \& Doyle, 2016; Jafari et al., 2008; Mohan \& Narsimhan, 1997; Raikar, Bhatia, Malone, \& Henson, 2009). Recent research in our laboratory has shown that stable concentrated emulsions can be produced by dual-channel high-pressure microfluidization using certain types of synthetic and natural emulsifier (Bai, Huan, Gu, \& McClements, 2016; Bai \& McClements, 2016a, 2016b). The current study focused on the potential of using a combination of two biosurfactants (rather than a single one) for forming and stabilizing highly concentrated emulsions. In particular, it was hypothesized that the utilization of mixed biosurfactants would improve the physical and chemical stability of the concentrated emulsions. The dual-channel homogenization method used in this study has rarely been used to form oil-in-water emulsions from natural emulsifiers, and so there is currently a relatively poor understanding of its capabilities. Nevertheless, it has some important advantages over conventional high-pressure methods for this purpose since it can continuously form emulsions in a single step, without the need to form an emulsion premix. Consequently, another important aim of this study was to elucidate the ability of dual-channel microfluidization to produce emulsions from mixed biosurfactants.

The selection of an appropriate emulsifier is one of the most important factors to consider when developing a successful emulsion-based product using any high-pressure homogenization method (Friberg et al., 2003; McClements, 2015; Stauffer, 1999). An emulsifier should rapidly adsorb to the droplet surfaces during homogenization, it should lower the interfacial tension to facilitate further droplet breakup, and it should form a protective coating to prevent droplet aggregation (Jafari et al., 2008; Santana, Perrechil, \& Cunha, 2013; Walstra, 1993). Moreover, there should be enough emulsifier present to cover the surfaces of all of the droplets formed during homogenization, otherwise droplet coalescence will occur inside the homogenizer (Tcholakova, Denkov, \& Danner, 2004). 
Many different kinds of emulsifiers are commercially available that can be used in food products, including polysaccharides, proteins, phospholipids, small molecule surfactants, and solid particles (Kralova \& Sjoblom, 2009; McClements, 2015; Stauffer, 1999). However, each type of emulsifier differs in its effectiveness at producing small droplets during homogenization, and in its ability to avoid droplet aggregation under different environmental conditions, such as $\mathrm{pH}$, ionic strength, heating, and freezing (Dickinson, 2003; Garti \& Reichman, 1993; McClements, 2015). Food emulsifiers also vary considerably in their cost, availability, ease of use, flavor profiles, ingredient compatibility, "label friendliness", and legal status (Kralova \& Sjoblom, 2009; Krog \& Sparso, 2004; Stauffer, 1999). Each type of emulsifier therefore has its own advantages and disadvantages that make it suitable for particular applications.

There is particular interest in the utilization of natural plant-based emulsifiers in food products because of their perceived "label friendliness" (Dickinson, 2003; Lam \& Nickerson, 2013; McClements \& Gumus, 2016; Ngouemazong, Christiaens, Shpigelman, Van Loey, \& Hendrickx, 2015). Quillaja saponins are biosurfactants that are highly effective at forming emulsions containing small droplets that are stable over a wide range of conditions (Ozturk, Argin, Ozilgen, \& McClements, 2015; Yang, Leser, Sher, \& McClements, 2013; Zhang, Bing, \& Reineccius, 2015, 2016). These biosurfactants have been reported to exhibit little or no toxicity at the levels used in foods (Kensil, Soltysik, Wheeler, \& Wu, 1996), but they do have a bitter taste when used at high concentrations (Ilsley, Miller, \& Kamel, 2005), which may limit their application in concentrated emulsions. Moreover, a recent study showed that it was difficult to produce highly concentrated emulsions (40 or $50 \%$ oil) by dual-channel microfluidization using quillaja saponins alone (Bai et al., 2016). The objective of the current study was therefore to determine whether concentrated emulsions containing small droplets could be formed using quillaja saponins in combination with another natural surfactant 
(lecithin). The influence of total biosurfactant concentration and biosurfactant ratio (saponin to lecithin) on the formation and stability of concentrated (50\% oil) oil-in-water emulsions produced by dual-channel microfluidization was therefore examined.

The results of this study are important for the development of highly concentrated emulsions produced from natural ingredients that can be used in food, beverage, and pharmaceutical applications.

\section{Materials and Methods}

\subsection{Materials}

De-oiled soy lecithin (Ultralec $\left.{ }^{\circledR} \mathrm{P}, \mathrm{DL}\right)$ was purchased from Archer Daniels Midland Co. (Decatur, IL), and was reported to contain $97 \mathrm{wt} \%$ lecithin. Quillaja saponin $\left(\mathrm{Q}-\mathrm{Naturale}^{\circledR}, \mathrm{QN}\right)$, which is an extract from the bark of the Quillaja saponaria tree, was kindly provided by Ingredion Inc. (Westchester, IL). Tween 80 (TN, MW 1310 Da, HLB 7), sodium phosphate monobasic and disodium hydrogen phosphate were provided by Sigma-Aldrich Co. (St. Louis, MO). Miglyol 812N, an example of a medium chain triglyceride (MCT), was purchased from Warner Graham Co. (Cockeysville, MD). All chemicals used were analytical grade. Double distilled and de-ionized water (Milli-Q) was used to prepare all solutions.

\subsection{Emulsion preparation}

Initially, an aqueous phase was prepared by weighing an amount of quillaja saponins or Tween $80(0.25$ to $2 \%(\mathrm{w} / \mathrm{w}))$ into a buffer solution $(5 \mathrm{mM}$ phosphate buffer, $\mathrm{pH} 7.0)$ and stirring for $2 \mathrm{~h}$ to ensure dissolution. The resulting aqueous solutions were then stored overnighted at $4{ }^{\circ} \mathrm{C}$. An oil phase was prepared by dispersing 0 or $1 \%(\mathrm{w} / \mathrm{w})$ of soy lecithin in MCT with heating at $50{ }^{\circ} \mathrm{C}$ for $30 \mathrm{~min}$, then stirring at ambient 
temperature for about $2 \mathrm{~h}$ to ensure complete dissolution. $50 \mathrm{wt} \%$ oil-in-water emulsions were then prepared using a dual-channel microfluidizer (Microfluidics PureNano, Newton, MA, USA). Simultaneously, the oil and aqueous phases were fed into the homogenizer using two separate glass reservoirs, and then forced to impinge upon each other under high pressure $(13,000$ psi or $89.6 \mathrm{MPa})$ using a series of narrow channels.

\subsection{Physical stability of emulsions}

Long-term storage and accelerated tests were used to determine the physical stability of the emulsions. Aliquots $(5 \mathrm{~mL})$ of emulsions to which $0.02 \mathrm{wt} \%$ of sodium azide was added as a preservative were dispensed into a series of test tubes. The samples were then stored at $25{ }^{\circ} \mathrm{C}$ in the dark for 7 days to determine their long-term stability under ambient quiescent conditions. The stability of the emulsions to phase separation was then determined by taking digital photographs. Physical stability tests were carried out for emulsions in the absence and presence of an oil soluble dye ( 5 Nile red $\mathrm{mg} / \mathrm{mL}$ ethanol), which was mixed with the emulsions after homogenization.

\subsection{Determination of particle size and $\zeta$ potential}

The particle size distribution of the emulsions was determined by static light scattering (Mastersizer 2000, Malvern Instruments Ltd., Malvern, Worcestershire, UK). Samples were diluted with buffer solution ( $5 \mathrm{mM}$ phosphate, $\mathrm{pH} 7.0)$ to ensure that light waves could pass through and to prevent multiple scattering effects. The mean particle size is reported as the surface-weighted $\left(d_{32}\right)$ mean diameter, which was calculated from the following equation:

$$
d_{32}=\sum n_{i} d_{i}^{3} / \sum n_{i} d_{i}^{2}
$$


Here, $n_{i}$ is the number of droplets of diameter $d_{i}$. An indication of the polydispersity of the droplets in the emulsions was obtained by calculating the span:

$$
\operatorname{Span}=\frac{d_{90}-d_{10}}{d_{50}}
$$

Here, $d_{10}, d_{50}$ and $d_{90}$ are the particle diameters at 10,50 and $90 \%$ cumulative volume, respectively. The $d_{90}-d_{10}$ term is a measure of the width of the particle size distribution, while $d_{50}$ is a measure of the median particle size.

The surface potential ( $\zeta$-potential) of the droplets in the emulsions was measured using an electrophoresis instrument (Zetasizer Nano-ZS90, Malvern Instruments, Worcestershire, UK). Samples were diluted 100-fold using buffer solution (5 mM phosphate, $\mathrm{pH}$ 7.0) and then transferred into a capillary test tube for measurement. Each sample was equilibrated for 60 second before data was collected and then the Smoluchowski equation was used to convert the measured mobilities into $\zeta$-potential values.

Reported values for the mean particle size and $\zeta$-potential measurements were calculated from two measurements made on two different samples.

\subsection{Microstructure analysis}

The microstructure of the emulsions was characterized using optical microscopy (Nikon Eclipse C1 80i, Nikon, Melville, NY, USA), with a $10 \times$ eyepiece and a $60 \times$ objective lens. For confocal microscopy, all the samples were dyed with Nile Red (a hydrophobic fluorescent dye) dissolved in ethanol $(5 \mathrm{mg} / \mathrm{mL}$ ethanol) so that the final emulsions contained about $0.017 \%$ of Nile red and $3.3 \%$ ethanol. This relatively low level of ethanol would not be expected to have a major impact on emulsion properties (Zeeb, Herz, McClements, \& Weiss, 2015). The excitation and emission wavelengths used to analyze the Nile Red were $543 \mathrm{~nm}$ and $605 \mathrm{~nm}$, respectively. All images were 
taken and analyzed using the instrument software program (EZ-CS1 version 3.8, Nikon, Melville, NY).

\subsection{Shear viscosity measurements}

The apparent shear viscosity of emulsions was characterized using a dynamic shear rheometer with a temperature-controlled concentric cylinder measurement cup (Kinexus, Malvern, Worcestershire, UK). Initially, emulsions were transferred into the temperature-controlled measurement cup, and then allowed to equilibrate to the required temperature $\left(25^{\circ} \mathrm{C}\right)$ prior to making measurements. Finally, the viscosity of the samples was determined at a constant shearing rate $\left(10 \mathrm{~s}^{-1}\right)$ for $3 \mathrm{~min}$, so that the samples could be compared under fixed conditions. Reported apparent shear viscosities were calculated from measurements made on four different samples.

\subsection{Equilibrium interfacial tension measurements}

The equilibrium interfacial tension versus surfactant concentration profile was measured at an oil-water interface using a drop shape analysis instrument (DSA 100, Kruss GmbH, Hamburg Germany). The oil phase consisted of either MCT oil or MCT oil containing $1 \%(\mathrm{w} / \mathrm{w})$ soy lecithin. Initial experiments were carried out to define the time when the interfacial tension reached a fairly constant value (around $15 \mathrm{~min}$ ). The quillaja saponin and Tween 80 concentration range used was from $0.0005 \%$ to $2.0 \%$ because over $2.0 \%$ the saponin solutions were too turbid to make reliable measurements. Reported interfacial tension values were calculated from measurements made on four different samples.

The surface pressure $(\pi)$ was calculated from the interfacial tension $(\gamma)$ data using the following equation: $\pi=\gamma_{0}-\gamma$, where $\gamma$ and $\gamma_{0}$ are the interfacial tensions measured in the presence and absence of surfactant. 


\subsection{Statistical analysis}

All data are shown as the mean \pm standard deviation (SD) of measurements made on two to four freshly prepared samples, with multiple measurements made per individual sample (see previous sections for details for each analytical method). Statistical comparisons were performed using Student's t-test with SPSS 16 software. $P$-values lower than 0.05 were considered to be significant.

\section{Results and discussion}

\subsection{Impact of emulsifier type and concentration on emulsion formation}

\subsubsection{Particle size and microstructure}

Initially, the impact of hydrophilic emulsifier concentration on the size of the droplets in $50 \mathrm{wt} \%$ MCT oil-in-water emulsions produced using the dual-channel microfluidizer under fixed homogenization conditions $(13,000$ psi or $89.6 \mathrm{MPa}, 1$ pass $)$ was determined using light scattering (Figs. 1 and 2) and confocal fluorescence microscopy (Fig. 3). These emulsions were prepared in the absence or presence of hydrophobic emulsifier (1\% lecithin) in the oil phase.

In the absence of hydrophobic emulsifier, there was a significant decrease in mean droplet diameter $\left(d_{32}\right)$ with increasing hydrophilic emulsifier concentration from 0.25 to $0.5 \mathrm{wt} \%$ (Figs. 1a and 1b). Moreover, the confocal fluorescence microscopy images indicated that the size of the individual oil droplets in the emulsions decreased appreciably with increasing hydrophilic emulsifier concentration (Fig. 3). The observed decrease in droplet size can be attributed to two physiochemical phenomena: (i) more droplet surface area can be stabilized at higher emulsifier levels; (ii) the droplet surfaces are covered more rapidly at higher emulsifier concentrations, thereby leading to less 
coalescence inside the homogenizer (Tcholakova et al., 2004). Above 0.5 wt\% hydrophilic emulsifier, the mean droplet diameter did not change appreciably as the emulsifier level was increased further, which can be attributed to the fact that the droplet size was mainly limited by the maximum disruptive energy generated by the homogenizer rather than by the emulsifier concentration (Jafari et al., 2008).

In the presence of hydrophobic emulsifier (lecithin) in the oil phase, there was also a decrease in mean droplet diameter with increasing hydrophilic emulsifier concentration for both systems (Figs. 1 and 3). However, at the same level of hydrophilic emulsifier, the mean particle diameters were appreciably smaller in the presence of the hydrophobic emulsifier. For example, at $2 \%$ quillaja saponin, the mean droplet diameter $\left(d_{32}\right)$ was 0.38 $\mu \mathrm{m}$ in the presence of $1 \%$ lecithin, but $0.74 \mu \mathrm{m}$ in its absence (Fig. 1a). Moreover, the microscopy images indicated that the oil droplets were smaller and more evenly dispersed throughout the system in the presence of lecithin (Fig. 3). The presence of the lecithin also reduced the polydispersity (span) of the emulsions, particularly at the lower hydrophilic emulsifier levels used (Figs. 2a and 2b). Overall, these results indicate that the utilization of both a hydrophilic and hydrophobic emulsifier improved the efficiency of the homogenization process leading to smaller droplets with narrower particle size distributions. This may have been because the lecithin molecules also adsorbed to the droplet surfaces during homogenization where they reduced the interfacial tension (thereby facilitating droplet fragmentation) and formed a protective layer (thereby reducing coalescence) (Kralova \& Sjoblom, 2009).

\subsubsection{Particle charge characteristics}

The impact of emulsifier type and concentration on the surface potential ( $\zeta$-potential) of the droplets in the emulsions was measured to obtain some insight into their interfacial compositions (Fig. 4). Moreover, the surface potential of emulsion droplets plays an 
important role in determining the stability, physicochemical properties, and functional attributes of emulsions.

In the absence of hydrophobic emulsifier, the $\zeta$-potential of the droplets in the emulsions stabilized by quillaja saponins was highly negative (-70 to $-76 \mathrm{mV})$, which can be attributed to anionic functional groups on the saponin molecules (Stanimirova et al., 2011). The magnitude of the negative charge increased slightly with increasing emulsifier concentration, which suggests that the saponins may have packed more densely at higher concentrations, thereby leading to a higher surface charge density. Conversely, the droplets in the emulsions stabilized by Tween 80 only had a moderate negative charge (-9 to $-10 \mathrm{mV}$ ) that did not depend strongly on emulsifier concentration. Previous studies have also reported that oil droplets coated by non-ionic surfactants can have a negative charge (Chang \& McClements, 2016), which may be attributed to the presence of anionic impurities in the oil or surfactant (such as free fatty acids) or to preferential adsorption of anionic hydroxyl ions $\left(\mathrm{OH}^{-}\right)$from the water to the lipid droplet surfaces (McClements, 2015).

The presence of the hydrophobic emulsifier (lecithin) in the oil phase prior to homogenization had a pronounced influence on the electrical characteristics of the oil droplets in the emulsions containing both types of hydrophilic emulsifier (Fig. 3). The $\zeta$-potential of the droplets in the emulsions containing only lecithin (1 wt $\%)$ was $-78.5 \pm$ $1.4 \mathrm{mV}$, which can be attributed to the presence of anionic phospholipids such as phosphatidylserine and phosphatidylglycerol (Li et al., 2015). At the lowest saponin concentration $(0.25 \%)$, the $\zeta$-potential of the droplets was more highly negative in the presence of lecithin $(-76.5 \mathrm{mV})$ than in its absence $(-70.0 \mathrm{mV})$. This suggests that some of the phospholipid molecules were present at the oil droplet surfaces, and that they were more negatively charged than the saponins. As the saponin concentration was increased in the emulsions containing lecithin, the droplets became less highly negatively charged, 
suggesting that there was a change in interfacial composition. Presumably, there was an increasing number of saponin molecules present at the oil droplet surfaces when higher levels were present in the emulsions. At the lowest Tween 80 level, the $\zeta$-potential was much more negative in the presence of lecithin $(-63.0 \mathrm{mV})$ than in its absence $(-8.4 \mathrm{mV})$, which again suggests that anionic phospholipid molecules accumulated at the oil droplet surfaces. Commercial lecithin contains anionic phospholipids, such as phosphatidylserine and phosphatidylglycerol, which lead to lecithin-coated oil droplets with negative charges (Li et al., 2015). There was a slight decrease in the magnitude of the negative charge as the Tween 80 concentration in the emulsions was increased (Fig. 3), suggesting that some of the phospholipids were displaced from the droplet surfaces by non-ionic surfactant molecules.

\subsection{Impact of emulsifier type and concentration on emulsion stability}

In commercial applications it is important that emulsions remain stable throughout their shelf-life (McClements, 2015). For this reason, the impact of emulsifier type and concentration on the storage stability of the concentrated emulsions was measured. The particle size, surface potential, and microstructure of the emulsions were measured using the same methods as described in the previous section for the initial emulsions. After 7-days storage, the mean particle size, particle charge, and microstructure of the emulsions followed similar general trends as the initial emulsions (see Supplementary Material), which suggested that most of the emulsions were fairly stable to droplet aggregation (e.g., coalescence or flocculation). Presumably, the repulsive interactions generated by the interfacial coatings (steric and electrostatic) were strong enough to overcome any attractive interactions (van der Waals) between the droplets (Hunter, 2001). Having said this, there was a substantial increase in the mean particle diameter in the emulsions containing no hydrophobic emulsifier and low hydrophilic emulsifier levels 
(see Supplementary material), which suggested that these emulsions were unstable to droplet aggregation. This is probably because they initially contained relatively large droplets, and were therefore more prone to coalescence (Demiere, Colin, Leal-Calderon, \& Bibette, 1998; Kabalnov, 1998).

Information about the gravitational separation of the emulsions during storage was obtained by taking digital photographs of them in the absence and presence of Nile red (Fig. 5). Nile red is an oil-soluble dye that can highlight the spatial location of oil droplets within an emulsion. In the absence of hydrophobic emulsifier, a distinct layer of oil was observed on top of the emulsions prepared using quillaja saponins at the lowest levels used $(0.25$ and $0.5 \%)$, which suggested that some droplet coalescence and oiling-off occurred. The rate of droplet coalescence is known to increase with increasing droplet size (Demiere et al., 1998; Kabalnov, 1998; McClements, 2015), and therefore may have occurred in these systems because they initially contained relatively large droplets. In addition, a cream layer was observed at the top of the emulsions prepared using both types of hydrophilic emulsifiers at low levels, which suggested that droplet creaming occurred in these systems. This effect can be attributed to the relatively large dimensions of the droplets in these emulsions, since the creaming velocity is proportional to the square of the particle diameter (McClements, 2015). As the concentration of the hydrophilic emulsifier used to prepare the emulsions was decreased the extent of creaming and oiling off increased. Interestingly, phase separation was difficult to detect in the non-dyed emulsions at high hydrophilic emulsifier levels, but was clearly seen in the dyed emulsions. Creaming is often difficult to detect in concentrated emulsions because the lightness does not change appreciably when the droplet content is increased from around 5 to 50\% (Chantrapornchai, Clydesdale, \& McClements, 1998, 1999). Indeed, all of the emulsions exhibited some droplet creaming after 7-days storage, which can be attributed to the fact that they all contained a population of relatively large 
droplets that creamed rapidly.

The creaming velocity of an isolated spherical rigid particle in an ideal liquid can be described by Stokes' Law (Hunter, 1987):

$$
v_{\text {Stokes }}=-\frac{d^{2} g\left(\rho_{2}-\rho_{1}\right)}{18 \eta_{1}}
$$

Here, $d$ is the droplet diameter, $g$ is the gravitational constant, $\rho$ is the density, $\eta$ is the viscosity, and the subscripts 1 and 2 refer to the continuous and disperse phases, respectively. For a concentrated emulsion, this equation must be modified to take into account droplet-droplet interactions (McClements, 2015):

$$
v=v_{\text {Stokes }}\left(1-\frac{\phi}{\phi_{c}}\right)^{k \phi_{c}}
$$

Here $k$ and $\phi_{\mathrm{c}}$ are semi-empirical constants that depend on the nature of the colloidal dispersion. For oil-in-water emulsions, the following parameters have been reported: $k=$ 8 and $\phi_{\mathrm{c}}=0.585$ (McClements, 2015).

The stability of an emulsion to gravitational separation can therefore be estimated using the modified Stokes' Law. To a first approximation, $\phi=0.5, g=10 \mathrm{~m} \mathrm{~s}^{-2}, \rho_{1}=1000 \mathrm{~kg} \mathrm{~m}^{-3}$, $\rho_{2}=950 \mathrm{~kg} \mathrm{~m}^{-3}$ and $\eta_{1}=0.001 \mathrm{~Pa} \mathrm{~s}^{-1}$ for the oil-in-water emulsions used in this study. Thus, the creaming velocity would be around $0.002,0.01,0.04,0.2,0.9$ and $4 \mathrm{~mm}$ per week for droplets of diameter $0.2,0.5,0.1,2,5$ and $10 \mu \mathrm{m}$, respectively. This simple calculation indicates that the majority of the droplets in a highly-concentrated emulsion should have diameters below about $5 \mu \mathrm{m}$ to substantially slowdown creaming. A fraction of the droplets in the emulsions prepared in this study had diameters above this critical value (Fig. 3), and would therefore be susceptible to creaming. We prepared the emulsions using a single pass through the dual channel microfluidizer at a fixed homogenization pressure. In future studies, it may be useful to use more passes, a higher 
operating pressure, or a higher emulsifier concentration to produce smaller droplets that are more resistant to creaming.

The incorporation of lecithin into the oil phase prior to homogenization improved the stability of the emulsions to phase separation, i.e. there was less oiling off and slower creaming (Fig. 5). It was again relatively difficult to detect phase separation in the non-dyed emulsions, especially at the higher hydrophilic emulsifier levels, but creaming could easily be seen in the dyed emulsions. As mentioned earlier, it is difficult to observe phase separation in concentrated emulsions because their appearance (lightness) does not change appreciably when the droplet concentrations is increased from around 5 to $50 \%$ (McClements, 2015).

\subsection{Impact of emulsifier type and concentration on emulsion viscosity}

The rheology of emulsion-based food products is important because it influences their processing (mixing or flow through a pipe), functional properties (pouring or spreading) and sensory attributes (texture and mouthfeel) (McClements, 2015). The impact of emulsifier type and concentration on the apparent shear viscosities of the emulsions was therefore investigated at a fixed shearing rate $\left(10 \mathrm{~s}^{-1}\right)$ (Bai, Huan, $\mathrm{Li}, \&$ McClements, 2017; Mayer, Weiss, \& McClements, 2013). In all systems, there were an increase in shear viscosity with increasing hydrophilic emulsifier concentration (Figs. 6a and $6 \mathrm{~b})$. This viscosity increase may have occurred for a number of reasons. First, the adsorbed emulsifier layer contributes to the effective volume fraction of the dispersed phase, i.e., oil core + emulsifier layer (McClements, 2015). The volume fraction occupied by the emulsifier layer will have increased as the emulsifier concentration increased due to the decrease in mean droplet diameter, i.e., higher surface area (Figs. 1a and 1b). Second, the viscosity of concentrated emulsions is known to decrease as the 
polydispersity of the droplets increases because small droplets can fit into the gaps between large droplets (Luckham \& Ukeje, 1999). In our study, the polydispersity (span) of the emulsions decreased with increasing hydrophilic emulsifier concentration (Figs. 2a and $2 b$ ), which would therefore be expected to increase the viscosity. Third, the presence of any non-adsorbed surfactant micelles in the aqueous phase may have generated an osmotic attraction between the droplets, which led to droplet flocculation through a depletion mechanism (Bibette, Roux, \& Pouligny, 1992; McClements, 1994). Droplet flocculation is known to increase the viscosity of emulsions by increasing the effective volume fraction of the dispersed phase due to the water trapped between the oil droplets within the flocs (Dickinson \& Golding, 1997; Klinkesorn, Sophanodora, Chinachoti, \& McClements, 2004). However, this mechanism appears to be unlikely since droplet flocculation was not observed in the confocal microscopy images (Fig. 3).

At the same level of hydrophilic surfactant, the emulsions containing lecithin had substantially higher viscosities than the ones containing no lecithin (Fig. 6a). If the lecithin remained entirely within the oil droplets, then one would not expect it to have a major impact on emulsion viscosity, provided the droplet size remained the same. However, there are a number of physicochemical mechanisms that may account for this effect. First, the presence of the lecithin at the droplet surfaces may have altered the interfacial rheology. Theoretical and experimental studies have shown that the viscosity of concentrated emulsions increases as the interface becomes more viscous or elastic (Pal, 2011; Wulff-Perez, Torcello-Gomez, Martin-Rodriguez, Galvez-Ruiz, \& de Vicente, 2011). Second, the presence of the lecithin in the oil phase led to smaller oil droplets with narrower particle size distributions after homogenization, which may have increased the emulsion viscosity for the reasons discussed previously (Luckham \& Ukeje, 1999). Third, some of the lecithin molecules may have moved into the aqueous phase and formed vesicles, thereby increasing the effective disperse phase volume fraction of the 
system and increasing the emulsion viscosity (McClements, 2015). In some practical applications, it is advantageous to have a highly viscous emulsion to obtain particular functional attributes, such as texture or mouthfeel, whereas in other applications it may be better to have an emulsion with a lower viscosity. Consequently, the viscosity increase caused by the presence of the lecithin in the emulsion may be either beneficial or deleterious depending on the application.

\subsection{Interfacial characteristics}

Additional insights into the potential impact of emulsifier type and concentration on the formation and stability of the emulsions was obtained by characterizing their interfacial characteristics. The equilibrium interfacial tension versus hydrophilic emulsifier concentration was therefore measured in the absence and presence of hydrophobic emulsifier (Fig. 7a), and the surface pressure was calculated from this data (Fig. 7b).

In the absence of hydrophobic emulsifier, the interfacial tension decreased appreciably as the concentration of hydrophilic emulsifier increased for both quillaja saponins and Tween 80 , which indicated that the surfactant molecules adsorbed to the oil-water interface and screened the unfavorable molecular interactions between oil and water (Fig. 7a). The surface pressure increased steeply when the emulsifier concentration was increased from 0 to $0.1 \mathrm{wt} \%$, but then increased more gradually until it reached a relatively constant value at high emulsifier levels (Fig. 7b). This suggested that the emulsifiers saturated the oil-water interface at the higher concentrations used. There was an appreciable difference in the ability of the two hydrophilic emulsifiers to lower the interfacial tension. For example, the interfacial tension decreased more steeply with increasing quillaja saponin concentration than with increasing Tween 80 concentration, and a lower interfacial tension was reached at saturation for the quillaja saponins (5.4 
$\mathrm{mN} / \mathrm{m})$ than for the Tween $80(7.9 \mathrm{mN} / \mathrm{m})$. These results suggest the saponins have a higher thermodynamic affinity for the oil-water interface, and that they may pack more efficiently at the interface at saturation, thereby screening the unfavorable hydrophobic interactions between the oil and water phase more effectively (McClements, 2015).

At a fixed hydrophilic emulsifier concentration, the interfacial tension of the systems containing lecithin was appreciably less than those containing no lecithin (Fig. 7a). This suggests that the mixed emulsifiers packed more efficiently at the oil-water interface so as to reduce the thermodynamically unfavorable contact between oil and water phases (Guzman et al., 2016). Interestingly, the interfacial tension was very low $(<0.2 \mathrm{mN} / \mathrm{m})$ for the systems containing $1 \%$ lecithin and relatively high levels of saponin (>0.1 wt $\%$ ). Very low interfacial tensions would be expected to lead to the formation of very small droplets during homogenization since less disruptive forces are required to breakdown the droplets (Jafari et al., 2008). However, the mean droplet diameter of the emulsions formed using high levels of quillaja saponin were actually slightly larger than those formed using Tween 80 (Fig. 1). This effect may have been because the emulsifiers were not able to adsorb to the droplet surfaces fast enough inside the homogenizer (Schubert \& Engel, 2004), or due to differences in the rheology of the interfaces formed by different emulsifiers (Walstra, 1993).

The critical micelle concentration (CMC) of emulsifiers is often used to characterize and compare their emulsifying ability (Hunter, 2001). The CMC can be calculated from the interfacial tension versus logarithm of emulsifier concentration profiles by determining the intersect between linear plots extrapolated from high and intermediate emulsifiers concentrations (Varona, Martín, \& Cocero, 2009). In this study, the effective CMCs of the individual emulsifiers and emulsifier blends were estimated from the data shown in Fig. 7a: 0.02, 0.002, 0.03 and 0.003 wt\% for saponins only, Tween 80 only, saponins + lecithin, and Tween $80+$ lecithin, respectively. The values for the individual 
emulsifiers are in reasonable agreement with the reported values of $0.0022 \mathrm{wt} \%$ for Tween 80 (Mandal, Nair, \& Ramaswamy, 1988) and 0.01 to $0.07 \mathrm{wt} \%$ for quillaja saponins from various sources (Mitra \& Dungan, 1997). Interestingly, the mixed emulsifier samples exhibited better emulsifying ability than the single emulsifiers, even though the effective CMC of the mixed emulsifiers was higher than that of the corresponding single emulsifiers. This may have been because both the hydrophilic and hydrophobic emulsifiers competed for the oil-water interface, and therefore a higher concentration of hydrophilic emulsifier was required to saturate the interfaces in the presence of a hydrophobic emulsifier (Guzman et al., 2016). In other words, the hydrophilic emulsifier had to displace some of the hydrophobic emulsifier from the droplet surfaces before it could saturate the interfaces.

The natural emulsifiers used in this study are actually compositionally complex materials that contain a mixture of different surface active components. Consequently, the calculated CMC values should only be regarded as only rough estimates rather than accurate values associated with a specific well-defined molecular species. It should also be noted that the interfacial tension at the droplet surfaces during homogenization will be a dynamic value that depends on how quickly the emulsifier molecules adsorb to the droplet surfaces during homogenization. In practice, it is difficult to measure the kinetics of emulsifier adsorption under conditions that mimic the highly vigorous turbulent conditions that occur during homogenization.

Recent studies have shown that the rheology of the interfacial layers formed by natural emulsifiers may impact emulsion formation and stability. For example, a study of emulsifier mixtures (saponins and whey proteins) adsorbed at oil-water interfaces indicated that the interfacial rheology was strongly influenced by the molecular interactions between the emulsifiers, which in turn impacted the aggregation stability of the the droplets in oil-in-water emulsions (Bottcher, Keppler, \& Drusch, 2017). Another 
study reported that there was a correlation between the rheology of interfacial layers formed by various plant and animal proteins and the formation and stability of oil-in-water emulsions (Amine, Dreher, Helgason, \& Tadros, 2014). In future studies, it would be useful to use similar analytical techniques to characterize the properties of the lecithin-saponin systems used in our study.

\section{Conclusions}

This study has shown that concentrated oil-in-water emulsions stabilized by single and mixed natural emulsifiers can be fabricated using dual-channel microfluidization. The mean diameter and polydispersity of the droplets in the emulsions decreased with increasing hydrophilic emulsifier concentration, which can mainly be attributed to the fact that a higher level of emulsifier can cover a greater surface area. Surface potential measurements indicated that there was a change in interfacial composition as the level of hydrophilic emulsifier (saponins or Tween 80) in the aqueous phase was changed, and that some of the hydrophobic emulsifier (lecithin) was located at the droplet surfaces. The quillaja saponin produced much more highly negatively charged droplets than the Tween 80. Inclusion of a hydrophobic emulsifier in the oil phase and a hydrophilic emulsifier in the aqueous phase prior to homogenization, led to the formation of smaller and more stable oil droplets than only using a single hydrophilic emulsifier. This effect may be due to the fact that the mixed emulsifier system led to a lower interfacial tension, which would facilitate droplet disruption during homogenization. The relatively small size and narrow polydispersity of the droplets in the emulsions formed from the mixed emulsifiers led to emulsions with a higher viscosity. The mixed-emulsifier emulsions also had a higher stability to droplet aggregation and creaming than the single-emulsifier emulsions due to their small size. However, some creaming was still observed in the emulsions after they were stored for 7 days, which was attributed to the presence of a population of 
droplets that was relatively large $(d>5 \mu \mathrm{m})$. For commercial applications, it may therefore be necessary to develop additional strategies to improve the creaming stability of the emulsions, e.g., by carrying out repeated homogenization to reduce the fraction of large droplets present or to add a thickening agent to the aqueous phase to inhibit droplet movement.

Overall, these results have important implications for the rational design of highly concentrated emulsions suitable for use in food, cosmetic, pharmaceutical, and other commercial products.

\section{Acknowledgements}

Xiang Luo (CSC No.: 201508210193) would like to thank the Chinese Scholarship Council for support. This material was partly based upon work supported by the Cooperative State Research, Extension, Education Service, USDA, Massachusetts Agricultural Experiment Station (MAS00491) and USDA, NRI Grants (2013-03795 and 2014-67021). We also thank Jenny Tang for useful advice and discussions on this work, and DSM for providing financial support. 


\section{References}

Amine, C., Dreher, J., Helgason, T., \& Tadros, T. (2014). Investigation of emulsifying properties and emulsion stability of plant and milk proteins using interfacial tension and interfacial elasticity. Food Hydrocolloids, 39, 180-186.

Bai, L., Huan, S., Gu, J., \& McClements, D. J. (2016). Fabrication of oil-in-water nanoemulsions by dual-channel microfluidization using natural emulsifiers:

Saponins, phospholipids, proteins, and polysaccharides. Food Hydrocolloids, 61, 703-711.

Bai, L., Huan, S., Li, Z., \& McClements, D. J. (2017). Comparison of emulsifying properties of food-grade polysaccharides in oil-in-water emulsions: Gum arabic, beet pectin, and corn fiber gum. Food Hydrocolloids, 66, 144-153.

Bai, L., \& McClements, D. J. (2016a). Development of microfluidization methods for efficient production of concentrated nanoemulsions: Comparison of single-and dual-channel microfluidizers. Journal of colloid and interface science, 466, 206-212.

Bai, L., \& McClements, D. J. (2016b). Formation and stabilization of nanoemulsions using biosurfactants: Rhamnolipids. Journal of Colloid and Interface Science, 479, 71-79.

Bibette, J., Roux, D., \& Pouligny, B. (1992). Creaming of emulsions - the role of depletion forces induced by surfactant. Journal De Physique Ii, 2(3), 401-424.

Bottcher, S., Keppler, J. K., \& Drusch, S. (2017). Mixtures of Quillaja saponin and beta-lactoglobulin at the oil/water-interface: Adsorption, interfacial rheology and emulsion properties. Colloids and Surfaces A: Physicochemical and Engineering Aspects, 518, 45-56.

Chang, Y. G., \& McClements, D. J. (2016). Characterization of mucin - lipid droplet interactions: Influence on potential fate of fish oil-in-water emulsions under simulated gastrointestinal conditions. Food Hydrocolloids, 56, 425-433.

Chantrapornchai, W., Clydesdale, F., \& McClements, D. J. (1998). Influence of droplet size and concentration on the color of oil-in-water emulsions. Journal of Agricultural and Food Chemistry, 46(8), 2914-2920.

Chantrapornchai, W., Clydesdale, F., \& McClements, D. J. (1999). Influence of droplet characteristics on the optical properties of colored oil-in-water emulsions. Colloids and Surfaces a-Physicochemical and Engineering Aspects, 155(2-3), 373-382.

Demiere, B., Colin, A., Leal-Calderon, G., \& Bibette, J. (1998). Lifetime and destruction of concentrated emulsions undergoing coalescence In B. P. Binks (Ed.), Modern Aspects of Emulsion Science. Cambridge, UK: The Royal Society of Chemistry 
Dickinson, E. (2003). Hydrocolloids at interfaces and the influence on the properties of dispersed systems. Food hydrocolloids, 17(1), 25-39.

Dickinson, E., \& Golding, M. (1997). Rheology of sodium caseinate stabilized oil-in-water emulsions. Journal of Colloid and Interface Science, 191(1), 166-176.

Friberg, S., Larsson, K., \& Sjoblom, J. (2003). Food emulsions: CRC Press. Garti, N., \& Reichman, D. (1993). Hydrocolloids as food emulsifiers and stabilizers. Food Structure, 12(4), 3.

Gupta, A., Eral, H. B., Hatton, T. A., \& Doyle, P. S. (2016). Nanoemulsions: formation, properties and applications. Soft Matter, 12(11), 2826-2841.

Guzman, E., Llamas, S., Maestro, A., Fernandez-Pena, L., Akanno, A., Miller, R., Ortega, F., \& Rubio, R. G. (2016). Polymer-surfactant systems in bulk and at fluid interfaces. Advances in Colloid and Interface Science, 233, 38-64.

Hunter, R. J. (1987). Foundations of Colloid Science, vol. I. vol II, 992-1052.

Hunter, R. J. (2001). Foundations of Colloid Science: Oxford University Press.

Ilsley, S., Miller, H., \& Kamel, C. (2005). Effects of dietary quillaja saponin and curcumin on the performance and immune status of weaned piglets. Journal of Animal Science, 83(1), 82-88.

Jafari, S. M., Assadpoor, E., He, Y., \& Bhandari, B. (2008). Re-coalescence of emulsion droplets during high-energy emulsification. Food hydrocolloids, 22(7),

1191-1202.

Kabalnov, A. S. (1998). Coalescence in emulsions. In B. P. Binks (Ed.), Modern Aspects of Emulsion Science. Cambridge, UK: The Royal Society of Chemistry

Kensil, C. R., Soltysik, S., Wheeler, D. A., \& Wu, J.-Y. (1996). Structure/function studies on QS-21, a unique immunological adjuvant from Quillaja saponaria Saponins

Used in Traditional and Modern Medicine (pp. 165-172): Springer.

Klinkesorn, U., Sophanodora, P., Chinachoti, P., \& McClements, D. J. (2004). Stability and rheology of corn oil-in-water emulsions containing maltodextrin. Food

Research International, 37(9), 851-859.

Kralova, I., \& Sjoblom, J. (2009). Surfactants Used in Food Industry: A Review. Journal of Dispersion Science and Technology, 30(9), 1363-1383.

Krog, N. J., \& Sparso, F. V. (2004). Food emulsifiers: Their chemical and physical properties. Food emulsions, 12.

Lam, R. S. H., \& Nickerson, M. T. (2013). Food proteins: A review on their emulsifying properties using a structure-function approach. Food Chemistry, 141(2), 975-984.

Li, J., Wang, X., Zhang, T., Wang, C., Huang, Z., Luo, X., \& Deng, Y. (2015). A review on phospholipids and their main applications in drug delivery systems. Asian Journal of Pharmaceutical Sciences, 10(2), 81-98.

Lissant, K. (1966). The geometry of high-internal-phase-ratio emulsions. Journal of 
Colloid and Interface Science, 22(5), 462-468.

Luckham, P. F., \& Ukeje, M. A. (1999). Effect of particle size distribution on the rheology of dispersed systems. Journal of Colloid and Interface Science, 220(2), 347-356.

Mandal, A. B., Nair, B. U., \& Ramaswamy, D. (1988). Determination of the critical micelle concentration of surfactants and the partition coefficient of an electrochemical probe by using cyclic voltammetry. Langmuir, 4(3), 736-739.

Mayer, S., Weiss, J., \& McClements, D. J. (2013). Vitamin E-enriched nanoemulsions formed by emulsion phase inversion: factors influencing droplet size and stability. Journal of colloid and interface science, 402, 122-130.

McClements, D. J. (1994). Ultrasonic determination of depletion flocculation in oil-in-water emulsions containing a nonionic surfactant. Colloids and Surfaces a-Physicochemical and Engineering Aspects, 90(1), 25-35.

McClements, D. J. (2015). Food emulsions: Principles, practices, and techniques (Third ed.). Boca Raton, FL: CRC Press.

McClements, D. J., \& Gumus, C. E. (2016). Natural emulsifiers - Biosurfactants, phospholipids, biopolymers, and colloidal particles: Molecular and physicochemical basis of functional performance. Advances in Colloid and Interface Science, 234, 3-26.

Mitra, S., \& Dungan, S. R. (1997). Micellar properties of Quillaja saponin. 1. Effects of temperature, salt, and pH on solution properties. Journal of Agricultural and Food Chemistry, 45(5), 1587-1595.

Mohan, S., \& Narsimhan, G. (1997). Coalescence of protein-stabilized emulsions in a high-pressure homogenizer. Journal of Colloid and Interface Science, 192(1), $1-15$.

Muller, R. H., Harden, D., \& Keck, C. M. (2012). Development of industrially feasible concentrated $30 \%$ and $40 \%$ nanoemulsions for intravenous drug delivery. Drug Development and Industrial Pharmacy, 38(4), 420-430.

Ngouemazong, E. D., Christiaens, S., Shpigelman, A., Van Loey, A., \& Hendrickx, M. (2015). The Emulsifying and Emulsion-Stabilizing Properties of Pectin: A Review. Comprehensive Reviews in Food Science and Food Safety, 14(6), 705-718.

Osborn, H. T., \& Akoh, C. C. (2004). Effect of emulsifier type, droplet size, and oil concentration on lipid oxidation in structured lipid-based oil-in-water emulsions. Food Chemistry, 84(3), 451-456.

Ozturk, B., Argin, S., Ozilgen, M., \& McClements, D. J. (2015). Nanoemulsion delivery systems for oil-soluble vitamins: Influence of carrier oil type on lipid digestion and vitamin D-3 bioaccessibility. Food Chemistry, 187, 499-506.

Pal, R. (2011). Influence of interfacial rheology on the viscosity of concentrated emulsions. Journal of Colloid and Interface Science, 356(1), 118-122. 
Piorkowski, D. T., \& McClements, D. J. (2014). Beverage emulsions: Recent developments in formulation, production, and applications. Food Hydrocolloids, 42, 5-41.

Raikar, N. B., Bhatia, S. R., Malone, M. F., \& Henson, M. A. (2009). Experimental studies and population balance equation models for breakage prediction of emulsion drop size distributions. Chemical Engineering Science, 64(10), 2433-2447.

Santana, R. C., Perrechil, F. A., \& Cunha, R. L. (2013). High- and Low-Energy Emulsifications for Food Applications: A Focus on Process Parameters. Food Engineering Reviews, 5(2), 107-122.

Schubert, H., \& Engel, R. (2004). Product and formulation engineering of emulsions. Chemical Engineering Research and Design, 82(9), 1137-1143.

Stanimirova, R., Marinova, K., Tcholakova, S., Denkov, N. D., Stoyanov, S., \& Pelan, E. (2011). Surface Rheology of Saponin Adsorption Layers. Langmuir, 27(20), 12486-12498.

Stauffer, C. (1999). Emulsifiers. St. Paul, MN: USA: Eagan Press handbook series.

Tcholakova, S., Denkov, N. D., \& Danner, T. (2004). Role of surfactant type and concentration for the mean drop size during emulsification in turbulent flow. Langmuir, 20(18), 7444-7458.

Varona, S., Martín, Á., \& Cocero, M. J. (2009). Formulation of a natural biocide based on lavandin essential oil by emulsification using modified starches. Chemical Engineering and Processing: Process Intensification, 48(6), 1121-1128.

Walstra, P. (1993). Principles of emulsion formation. Chemical Engineering Science, 48(2), 333-349.

Wulff-Perez, M., Torcello-Gomez, A., Martin-Rodriguez, A., Galvez-Ruiz, M. J., \& de Vicente, J. (2011). Bulk and interfacial viscoelasticity in concentrated emulsions: The role of the surfactant. Food Hydrocolloids, 25(4), 677-686.

Yang, Y., Leser, M. E., Sher, A. A., \& McClements, D. J. (2013). Formation and stability of emulsions using a natural small molecule surfactant: Quillaja saponin (Q-Naturale (R)). Food Hydrocolloids, 30(2), 589-596.

Zeeb, B., Herz, E., McClements, D. J., \& Weiss, J. (2015). Reprint of: Impact of alcohols on the formation and stability of protein-stabilized nanoemulsions. Journal of

Colloid and Interface Science, 449, 13-20.

Zhang, J., Bing, L., \& Reineccius, G. A. (2015). Formation, optical property and stability of orange oil nanoemulsions stabilized by Quallija saponins. Lwt-Food Science and Technology, 64(2), 1063-1070.

Zhang, J., Bing, L., \& Reineccius, G. A. (2016). Comparison of modified starch and Quillaja saponins in the formation and stabilization of flavor nanoemulsions. Food Chemistry, 192, 53-59. 


\section{Figure captions}

Fig. 1. Influence of hydrophilic emulsifier type and concentration on the mean droplet diameters $\left(\mathrm{d}_{32}\right)$ of $50 \mathrm{wt} \%$ oil-in-water emulsions $(5 \mathrm{mM}$ phosphate buffer, $\mathrm{pH}$ 7.0) prepared by dualchannel microfluidization (13 kpsi, 1 pass) in the absence and presence of hydrophobic emulsifier (lecithin). The hydrophilic emulsifiers used were (a) quillaja saponins and (b) Tween 80.

Fig. 2. Influence of hydrophilic emulsifier type and concentration on the polydispersity (span) of 50 wt\% oil-in-water emulsions ( $5 \mathrm{mM}$ phosphate buffer, $\mathrm{pH}$ 7.0) in the absence and presence of hydrophobic emulsifier (lecithin). The hydrophilic emulsifiers used were (a) quillaja saponins and (b) Tween 80.

Fig. 3. Influence of hydrophilic emulsifier type and concentration on the microstructure of $50 \mathrm{wt} \%$ oil-in-water emulsions ( $5 \mathrm{mM}$ phosphate buffer, $\mathrm{pH} 7.0$ ) prepared by dual-channel microfluidization (13 kpsi, 1 pass) in the absence and presence of hydrophobic emulsifier (lecithin): (a) quillaja saponins; (b) Tween 80. Images were taken by confocal fluorescence microscopy, and the lipid phase was dyed red. The scale bars $(50 \mu \mathrm{m})$ are shown in the lower right hand corner of each image.

Fig. 4. Influence of hydrophilic emulsifier type and concentration on the surface potential (zpotential) of the droplets in 50 wt\% oil-in-water emulsions $(5 \mathrm{mM}$ phosphate buffer, $\mathrm{pH} 7.0$ ) in the absence and presence of hydrophobic emulsifier (lecithin): (a) quillaja saponins; (b) Tween 80.

Fig. 5. Influence of hydrophilic emulsifier type and concentration on the appearance of $50 \mathrm{wt} \%$ oil-in-water emulsions ( $5 \mathrm{mM}$ phosphate buffer, $\mathrm{pH} 7.0$ ) in the absence and presence of hydrophobic emulsifier (lecithin). The red stained regions represent lipids. The emulsions were stored for 7 days under ambient conditions $(25 \mathrm{o})$ ). The emulsions in the upper pictures contained an oil-soluble dye (Nile Red) to highlight the location of the lipid phase, while those in the lower picture were not labeled.

Fig. 6. Influence of hydrophilic emulsifier type and concentration on the apparent shear viscosity (at $10 \mathrm{~s}^{-1}$ ) of $50 \mathrm{wt} \%$ oil-in-water emulsions in the absence and presence of hydrophobic emulsifier (lecithin): (a) quillaja saponins; (b) Tween 80.

Fig. 7a. Influence of hydrophilic emulsifier type (saponin or Tween 8) and concentration on the equilibrium interfacial tension measured at an oil-water interface in the absence and presence of a hydrophobic emulsifier (1\% lecithin). The oil phase was MCT, while the aqueous phase was $5 \mathrm{mM}$ phosphate buffer $(\mathrm{pH} 7)$. Inset shows a typical oil droplet used to measure the interfacial tension by drop shape analysis. The interfacial tension for $1 \%$ lecithin alone was $17.00 \pm$ $0.66 \mathrm{mN} / \mathrm{m}$. 
Fig. 7b. Influence of hydrophilic emulsifier type (saponin or Tween 8) and concentration on the surface pressure measured at an oil-water interface in the absence and presence of a hydrophobic emulsifier (1\% lecithin). The oil phase was MCT, while the aqueous phase was $5 \mathrm{mM}$ phosphate buffer ( $\mathrm{pH} 7)$. 
Figure 1(a)

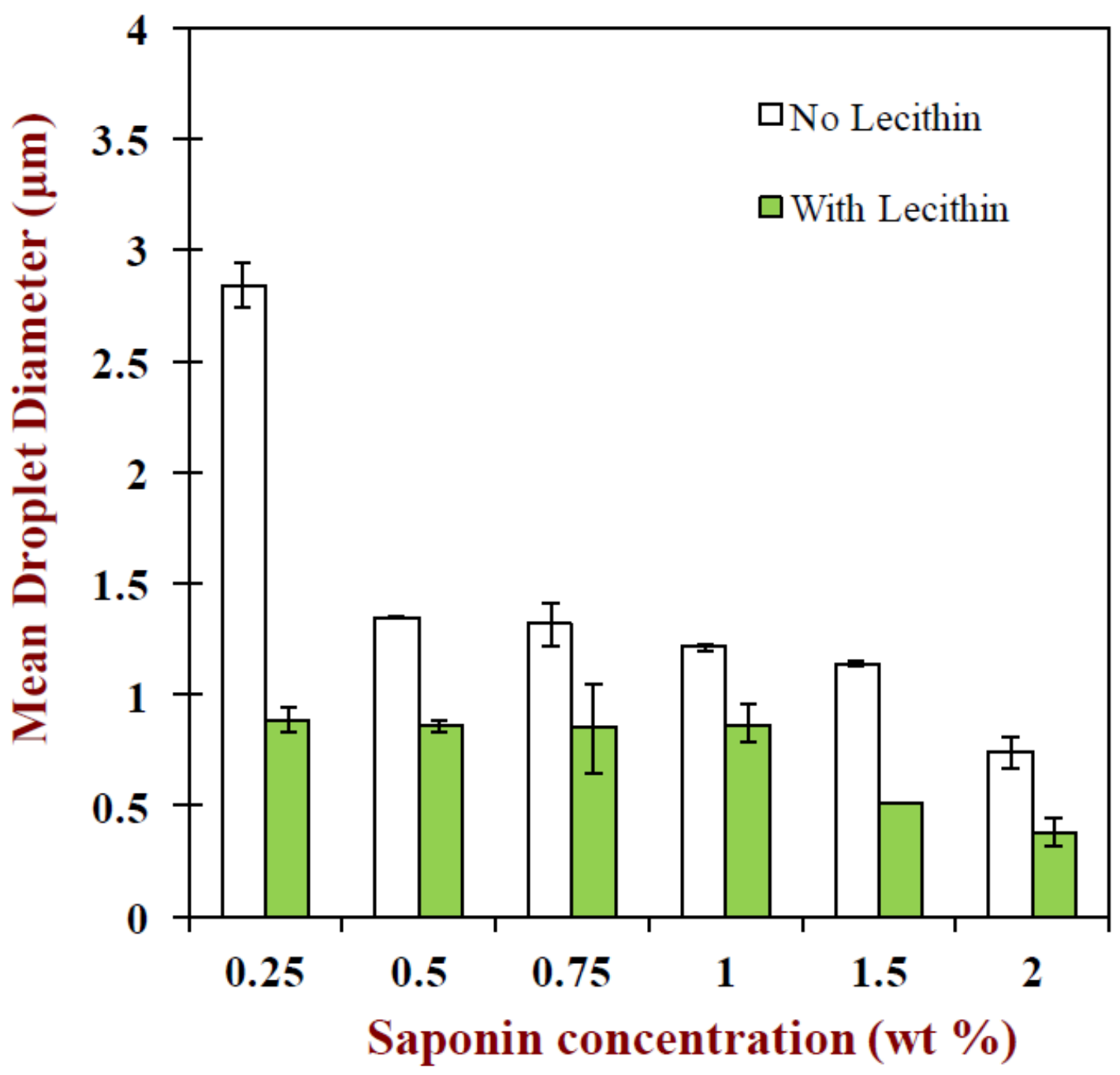


Fig $1 \mathrm{~b}$

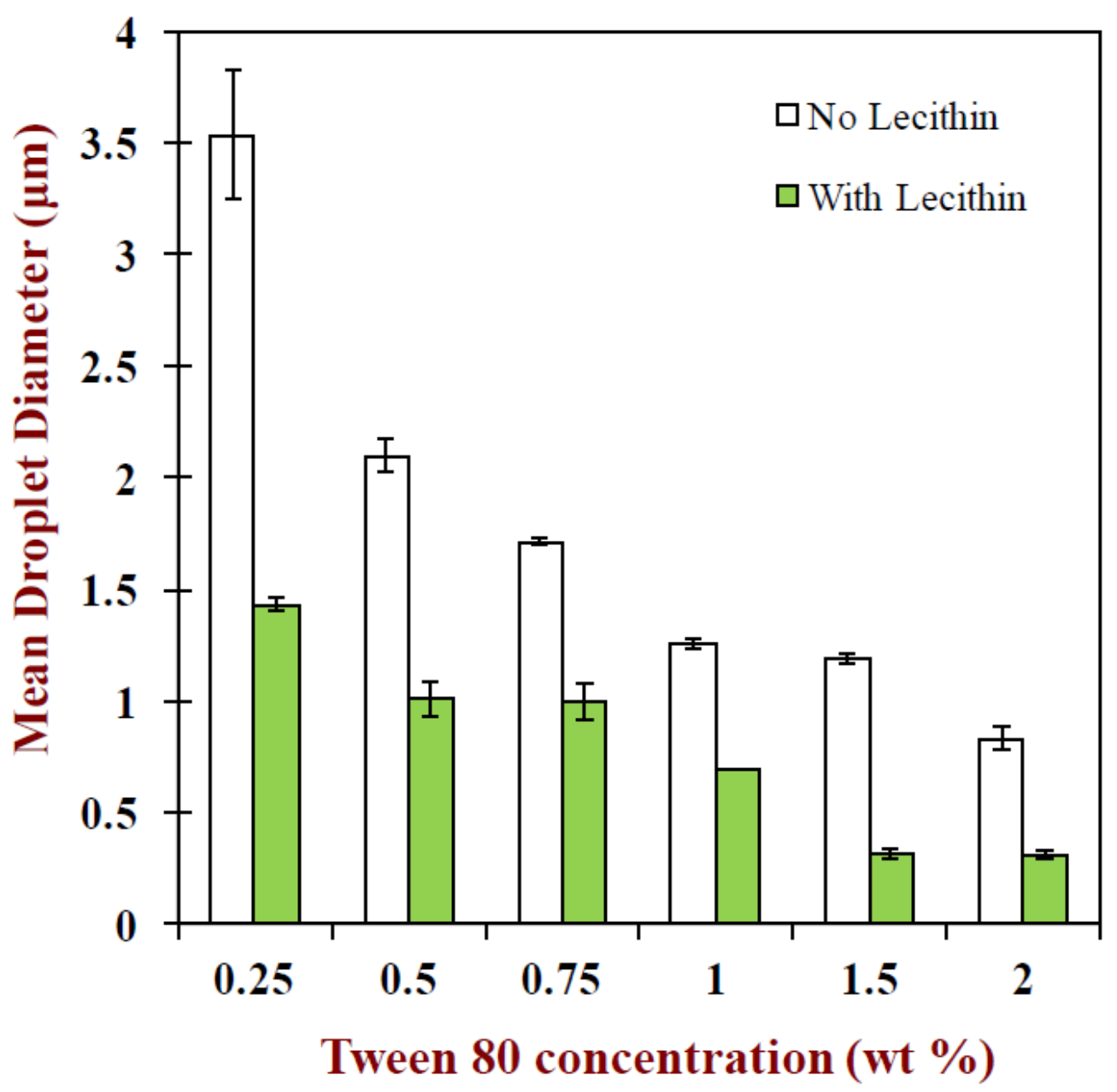


Figure 2a

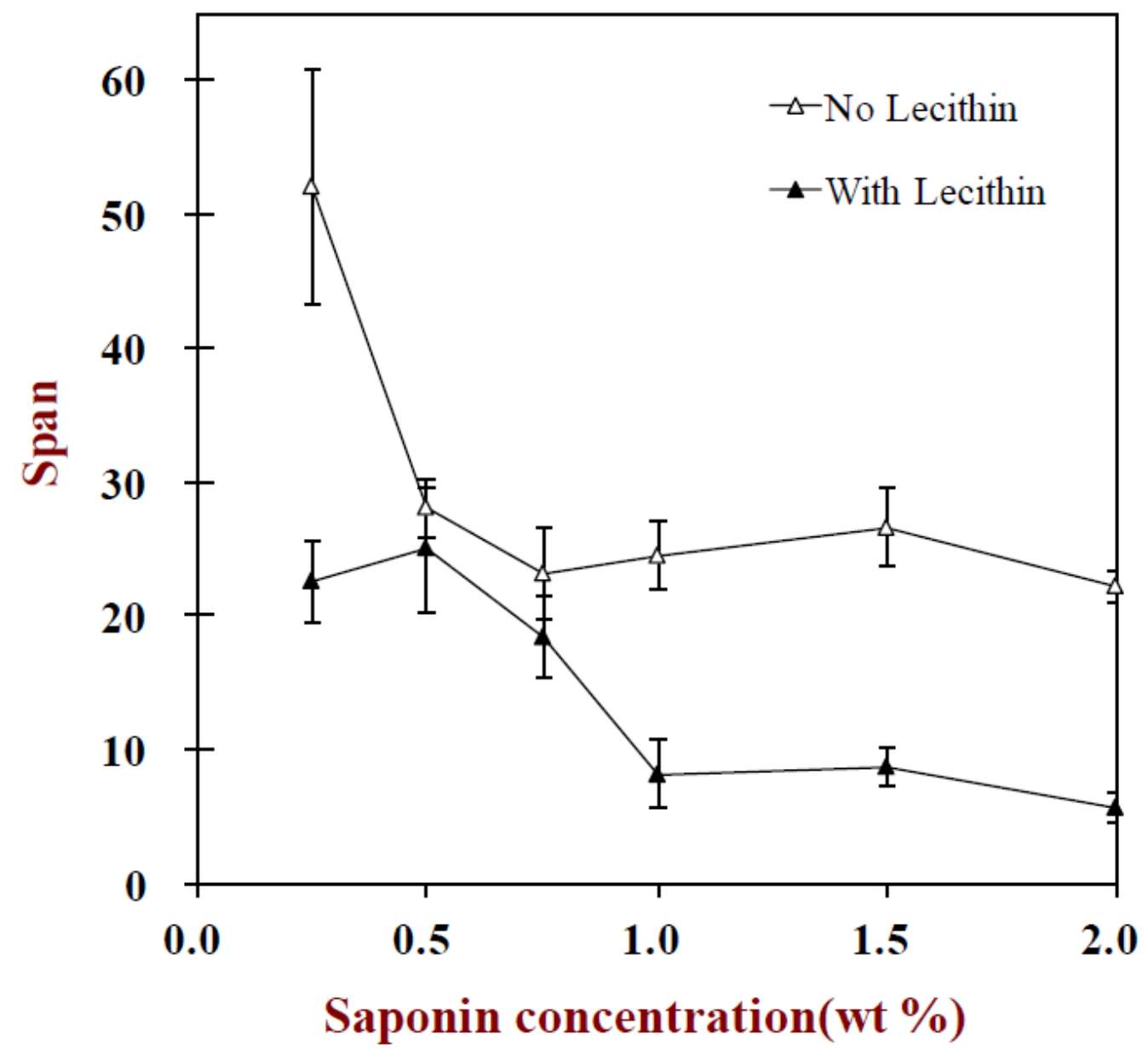


Figure 2b

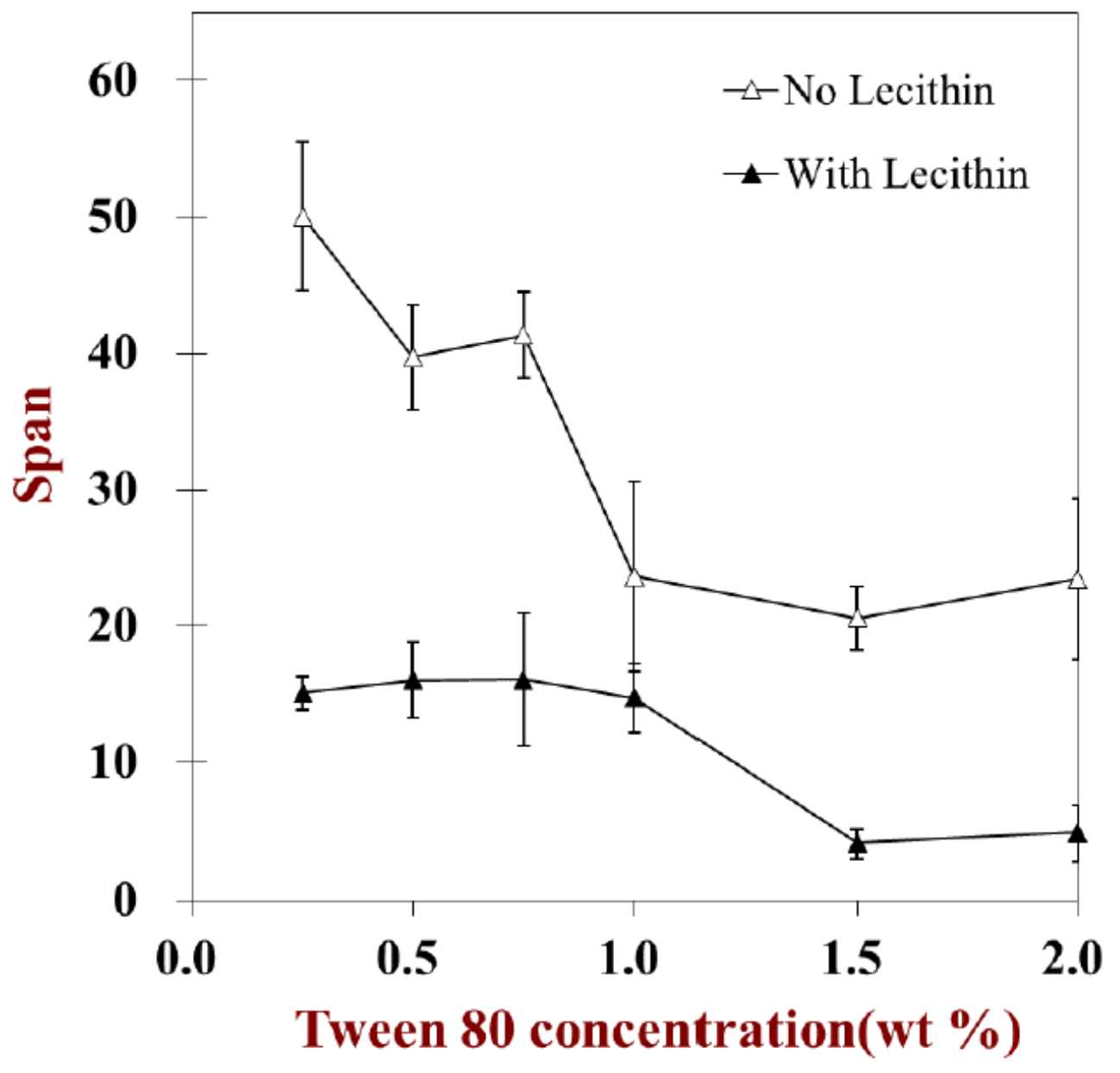


Figure 3

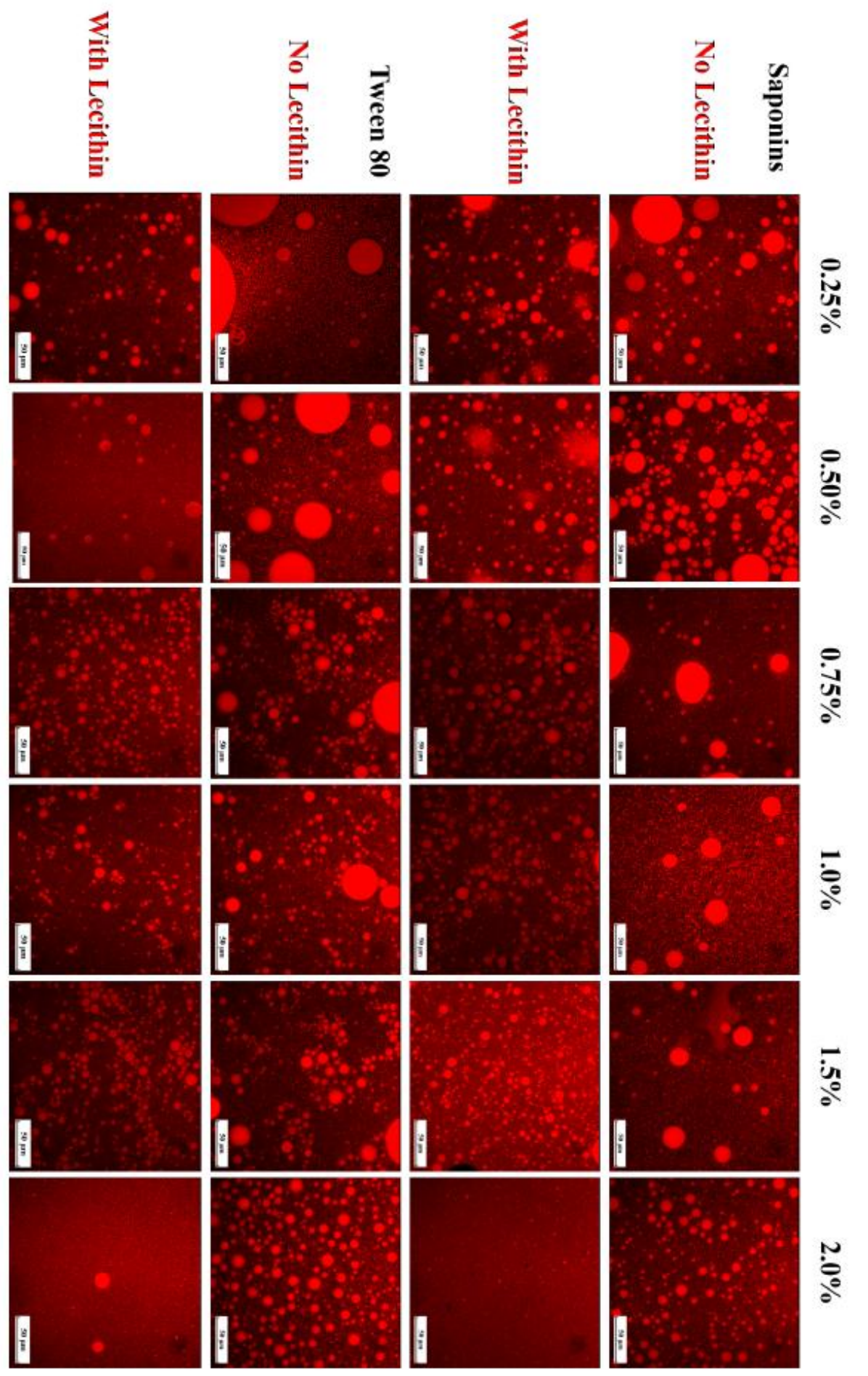


Figure 4a

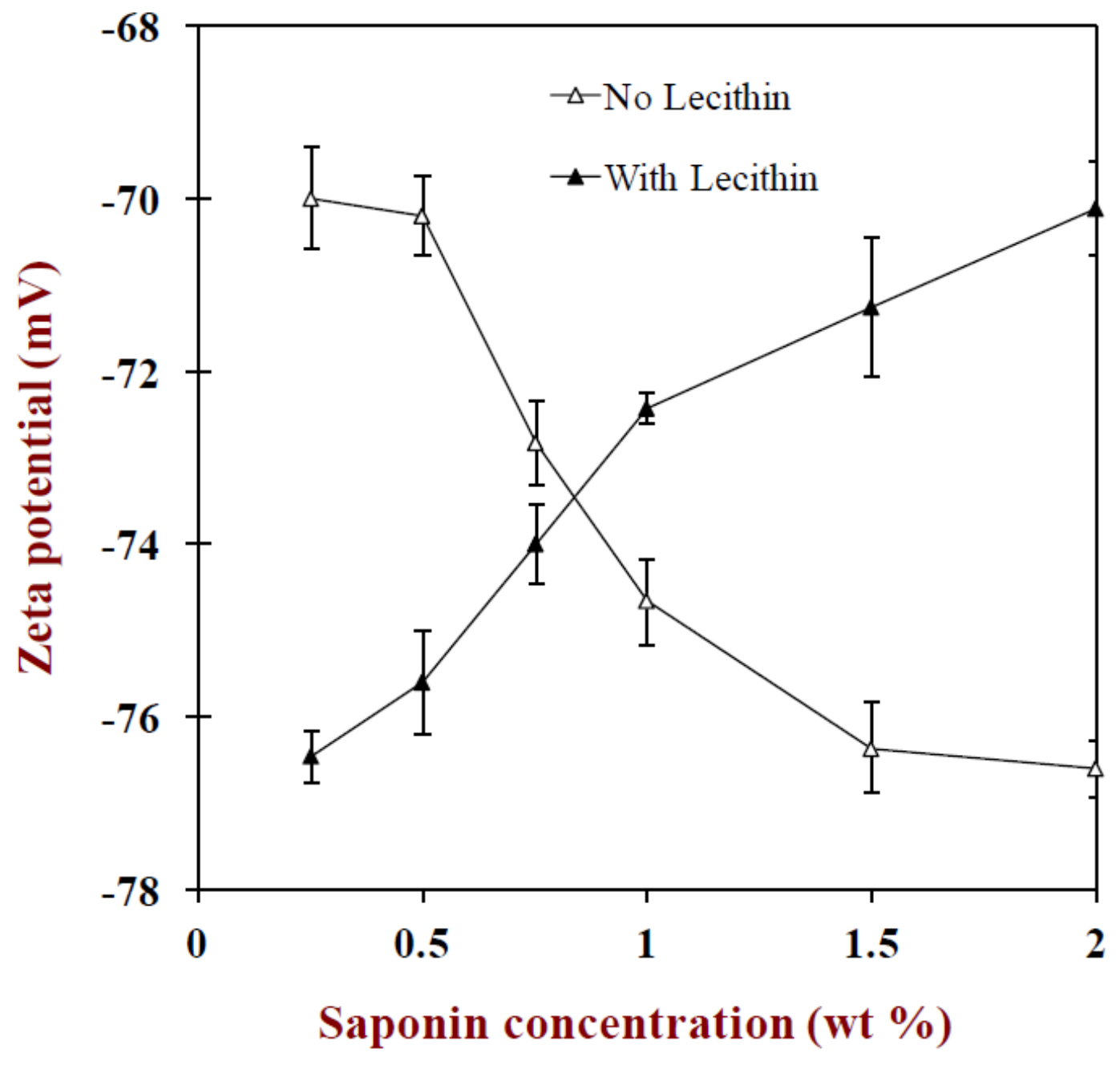


Figure $4 b$

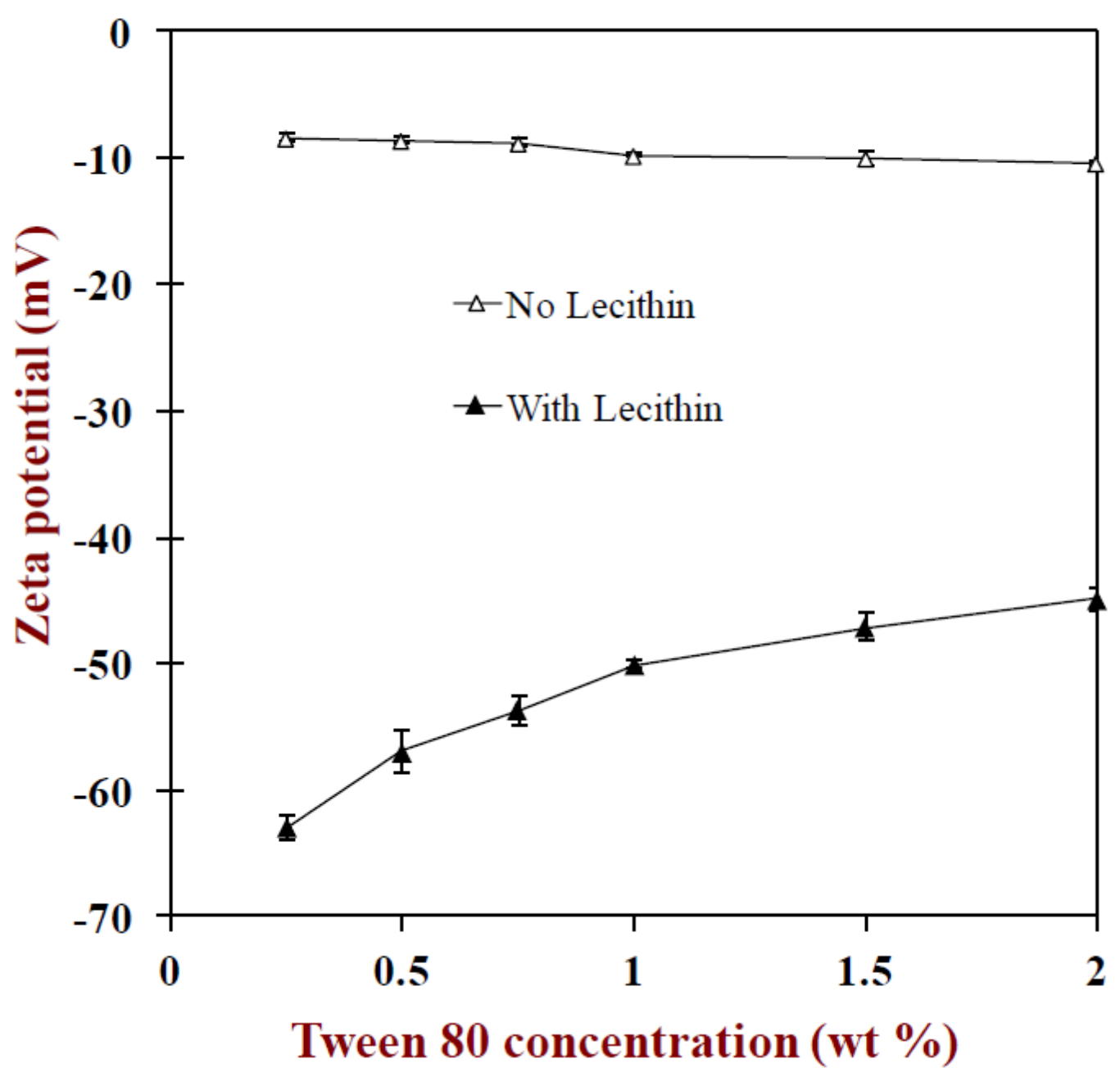


Figure 5

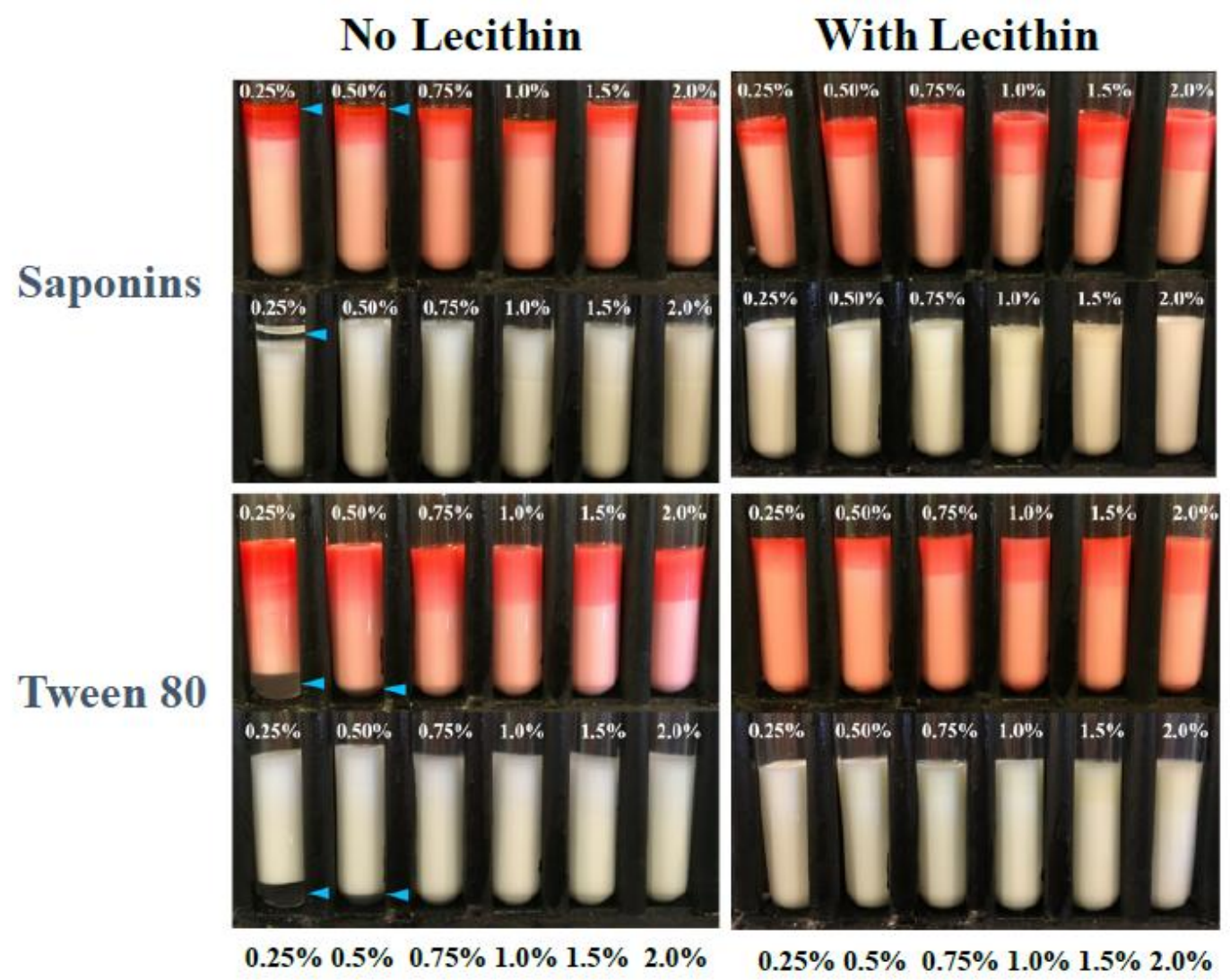


Fig 6a

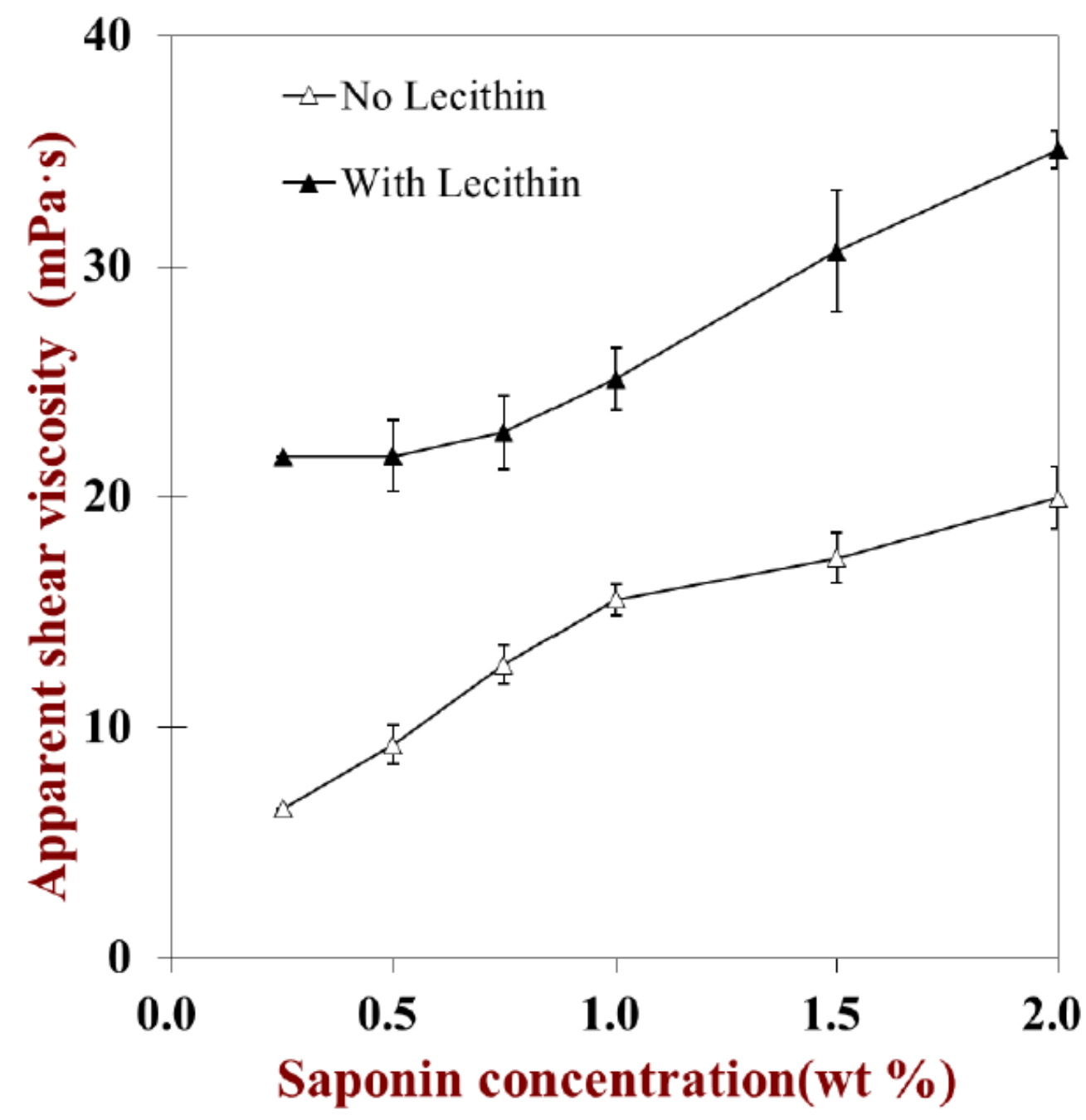


Fig $6 b$.

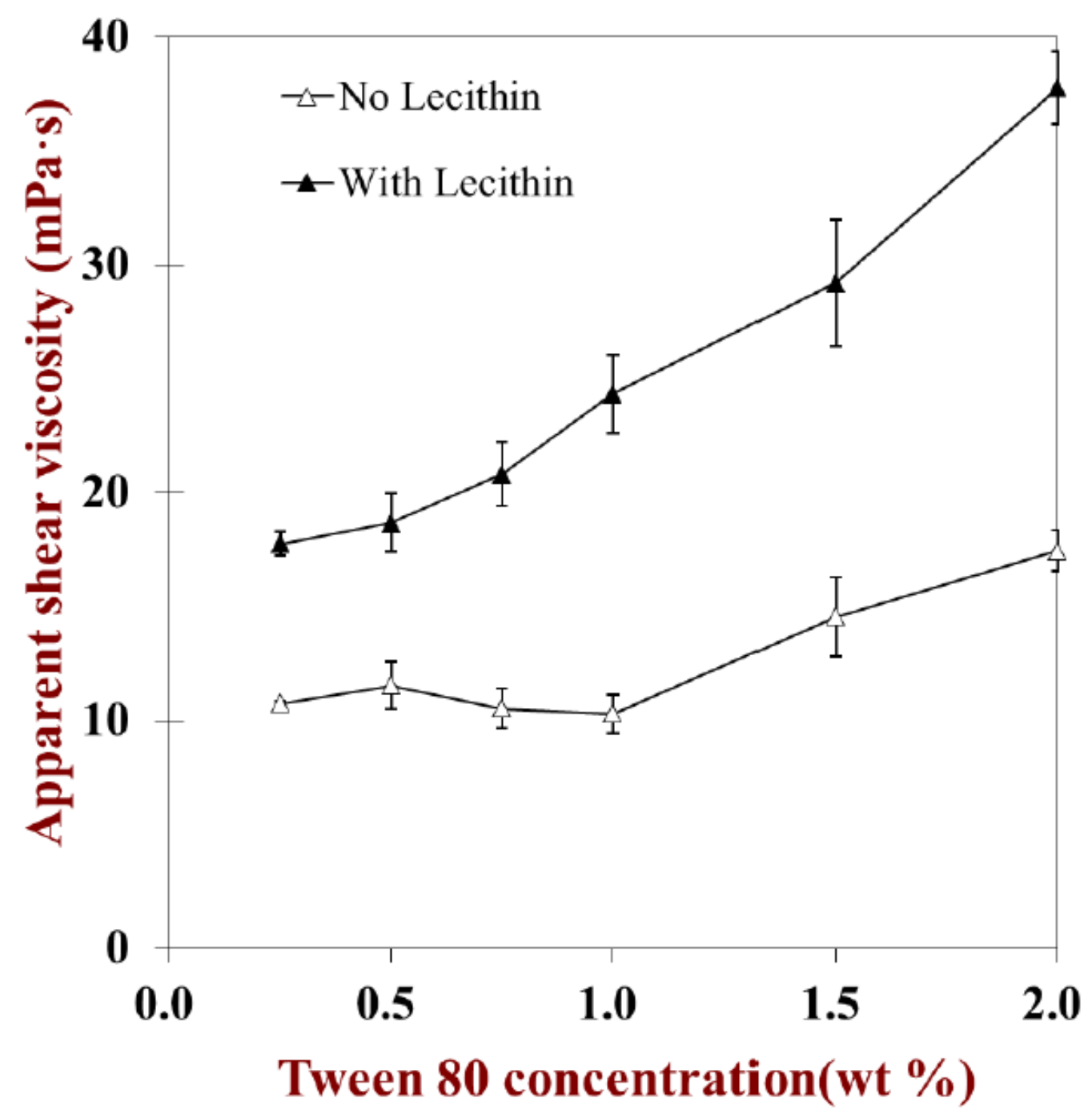


Figure 7a

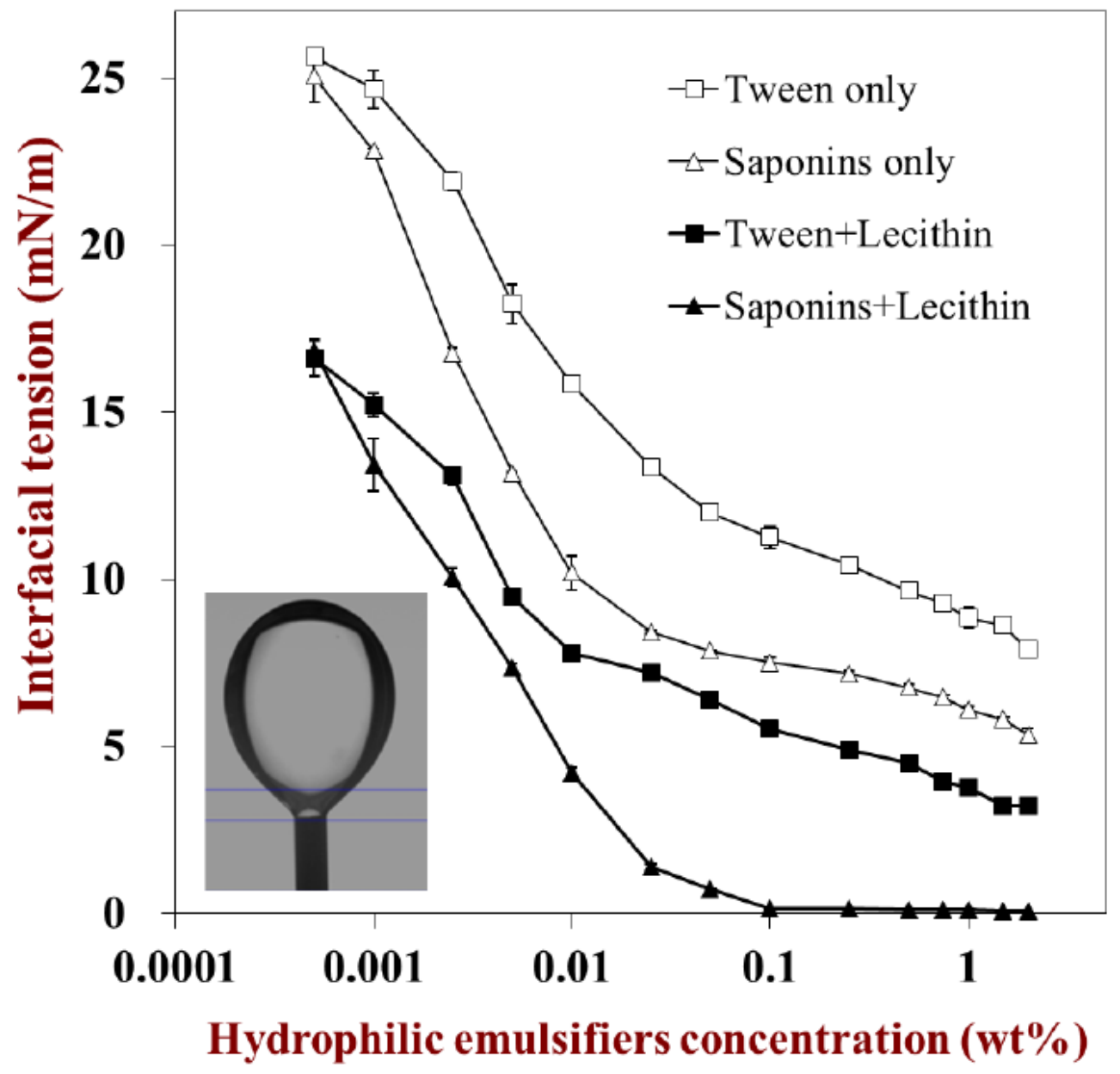


Figure $7 b$

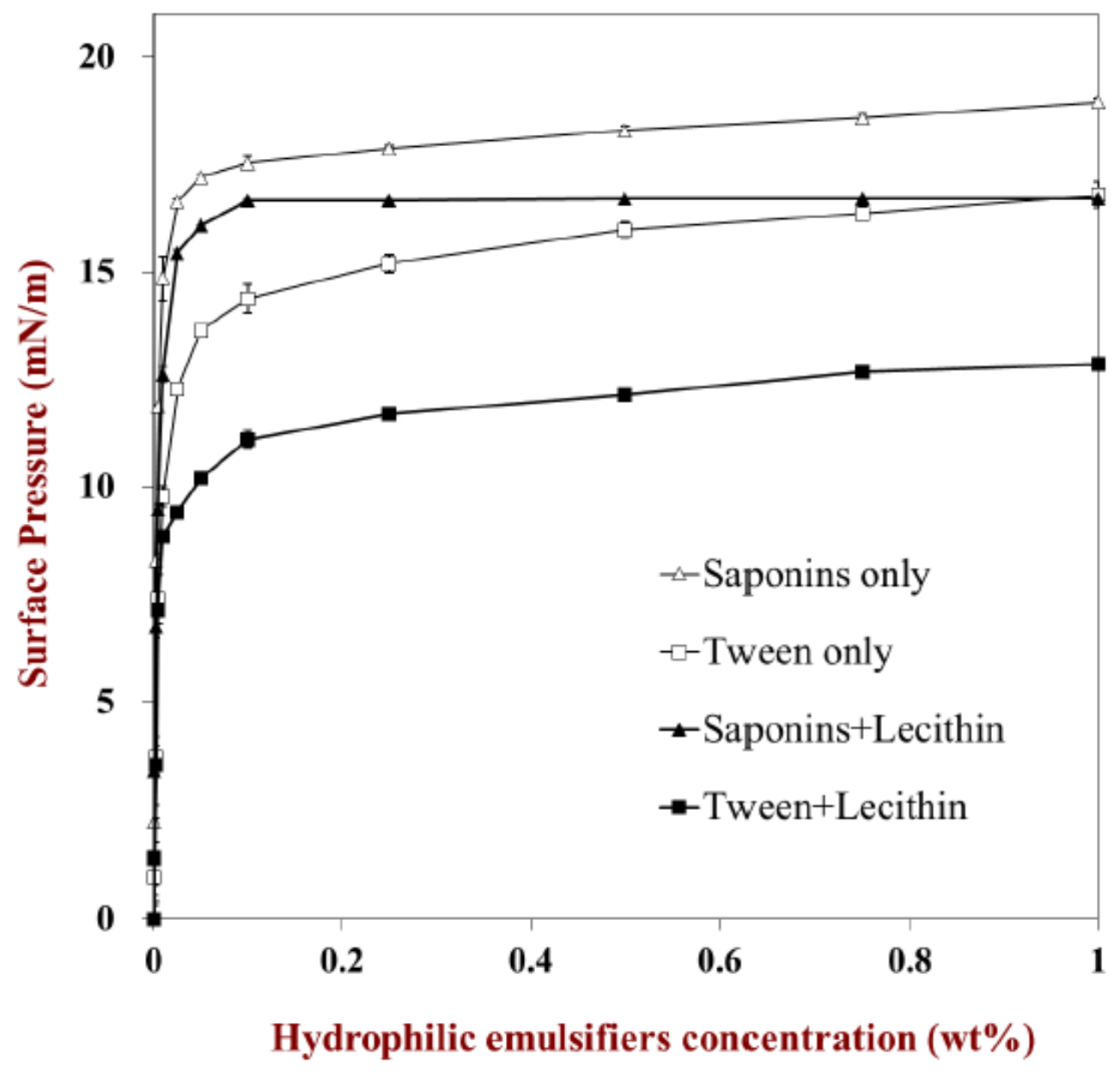




\section{Graphical Abstract}

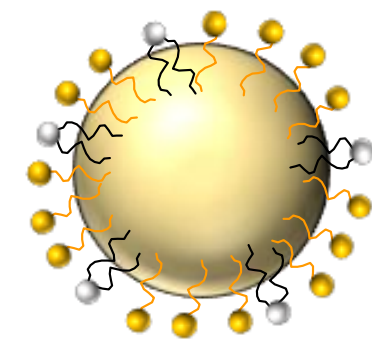

Mixed emulsifiers at droplet surfaces

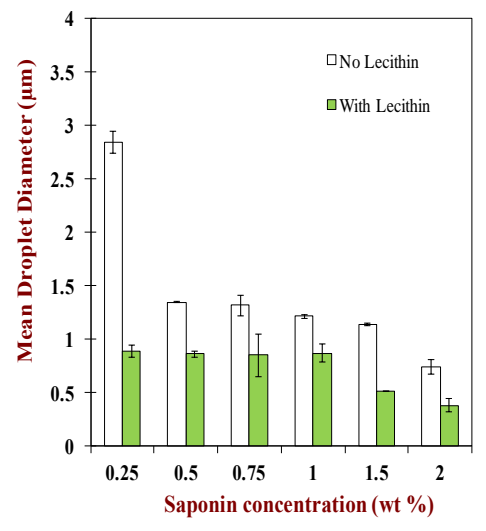

